EPJ manuscript No.

(will be inserted by the editor)

\title{
Neutron-proton mass difference in nuclear matter
}

\author{
Ulf-G. Meißner ${ }^{1,2}$, A. M. Rakhimov ${ }^{3}$, A. Wirzba ${ }^{2}$, and U. T. Yakhshiev ${ }^{2,4}$ \\ 1 Helmholtz-Institut für Strahlen- und Kernphysik (Theorie), D-53115, Universität Bonn, Germany \\ 2 Forschungszentrum Jülich, Institut für Kernphysik (Theorie), D-52425 Jülich, Germany \\ 3 Institute of Nuclear Physics, Academy of Sciences of Uzbekistan, Tashkent-132, Uzbekistan \\ 4 Physics Department \& Institute of Applied Physics, National University of Uzbekistan, Tashkent-174, Uzbekistan
}

Received: date / Revised version: date

\begin{abstract}
Isospin-breaking effects in nuclear matter are studied in the framework of a medium-modified Skyrme model. The proposed effective Lagrangian incorporates both the medium influence of the surrounding nuclear environment on the single nucleon properties and an explicit isospin-breaking effect in the mesonic sector. The approach predicts that the neutron-proton mass difference decreases in isospinsymmetric nuclear matter but by a very small amount only.
\end{abstract}

PACS. 12.39.Dc Skyrmions - 14.20.Dh Protons and neutrons - 21.65.+f Nuclear matter

\section{Introduction}

The evaluation of isospin-breaking effects in a dense nuclear medium is an interesting problem in nuclear physics. This is particularly the case for the Nolen-Schiffer anomaly 1 in mirror nuclei which may be explained by a change of the neutron-proton mass difference in the nuclear environment. Henley and Krein [2] argued that the NolenSchiffer anomaly can be resolved if the neutron-proton mass difference decreases rapidly in the medium. For this reason various approaches for calculating the neutron-proton mass difference in a dense nuclear environment have been proposed in the literature $[3,4,5,6,7,8,9$. Some of these approaches indeed confirmed a decrease [3,4,5, 6, 7. of the neutron-proton mass difference in the nuclear medium, while others gave opposite results [8,9]. Since the Skyrme model [10,11] treats the properties [12, 13, 14] and the interactions [15, 16, 17, 18, of the nucleons on an equal footing, it is interesting to study what this model has to say for possible medium-modifications of this quantity. Note also that the neutron-proton mass difference is composed of a strong and an electromagnetic contribution of comparable size, which might be affected differently in a dense medium.

In the present work we evaluate isospin-breaking effects in the baryonic sector of the Skyrme model by incorporating the influence of the surrounding nuclear environment on the nucleons as e.g. in Refs. [19,20. In order to perform our studies we modify the nonlinear $\sigma$-model Lagrangian, which is generalized by explicit isospin-breaking effects in the mesonic sector 21, based on the well-known pionic field equations in the nuclear medium 22 . Furthermore, the usual Skyrme term is added in order to stabilize the solitons of the model.
The paper is organized as follows. In Sec. 2 we discuss our model Lagrangian. Section 3 is devoted to the study of the field equations to be solved. After an explanation of the quantization procedure and the derivation of the strong part of the density-dependent neutron-proton mass difference in Sec. 4, we will present the electromagnetic (EM) part of that mass difference in terms of electromagnetic form factors in Sec. 5. The input values of the model parameters used in this work are discussed in Sec. 6. Section 7 is devoted to the presentation and discussion of the results. Finally, in Sec. 8 our conclusions are summarized and an outlook for further studies is given.

\section{Medium-modified Lagrangian}

We start from a generalized Lagrangian which incorporates an explicit isospin breaking term in the mesonic sector

$$
\begin{aligned}
\mathcal{L}= & \mathcal{L}_{2}+\mathcal{L}_{4}+\mathcal{L}_{\mathrm{g} \chi \mathrm{SB}}, \\
\mathcal{L}_{2}= & -\frac{F_{\pi}^{2}}{16} \operatorname{Tr} L_{\mu} L^{\mu}, \\
\mathcal{L}_{4}= & \frac{1}{32 e^{2}} \operatorname{Tr}\left[L_{\mu}, L_{\nu}\right]^{2}, \\
\mathcal{L}_{\mathrm{g} \chi \mathrm{SB}}= & -\frac{F_{\pi}^{2}}{16}\left\{\operatorname{Tr}(U-1) \mathcal{M}_{+}\left(U^{\dagger}-1\right) \mathcal{M}_{+}\right. \\
& \left.-\operatorname{Tr}(U-1) \mathcal{M}_{-} \tau_{3}\left(U^{\dagger}-1\right) \mathcal{M}_{-} \tau_{3}\right\},
\end{aligned}
$$

where $L_{\mu}=U^{\dagger} \partial_{\mu} U$. The Einstein summation convention for repeated Greek or Latin indices is adopted, unless stated otherwise. $\mathcal{L}_{2}$ is the usual Lagrangian of the nonlinear sigma model, $\mathcal{L}_{4}$ is Skyrme's stabilizing fourth order 
term. $\mathcal{L}_{\mathrm{g} \chi \mathrm{SB}}$ is a generalized pion-mass term proposed by Rathske [21] which includes explicitly isospin-breaking effects in the second term. Note that the latter has the same structure as the strong isospin-breaking fourth-order Lagrangian of chiral perturbation theory, see the term proportional to the low-energy constant $L_{7}$, Eq. (6.16) of Ref. 23. 11 Skyrme models with isospin-breaking terms proportional to the $\rho-\omega$ meson-mixing, as $e$.g. discussed in Refs. 24, 25, are actually of the same order in the chiral expansion, but more involved because of the additional meson degrees of freedom. The parameter $F_{\pi}$ is the pion decay constant, while $e$ is the dimensionless Skyrme constant. The $\mathrm{SU}(2)$ matrix $U(t, \boldsymbol{r})=\exp \left\{2 i \boldsymbol{\tau} \cdot \boldsymbol{\Phi}(t, \boldsymbol{r}) / F_{\pi}\right\}$ represents the isotriplet of pion fields $\boldsymbol{\Phi}$. Their masses, $m_{\pi^{ \pm}}$and $m_{\pi^{0}}$, enter the Lagrangian in the following combinations

$$
\mathcal{M}_{ \pm}=\sqrt{\left(m_{\pi^{ \pm}}^{2} \pm m_{\pi^{0}}^{2}\right) / 2} .
$$

The medium-modified Lagrangian, which takes into account the influence of the surrounding nuclear environment on the pion fields, can be set up by following the steps presented in Ref. [19. It has the form 2

$$
\begin{aligned}
\mathcal{L}^{*}= & \mathcal{L}_{2}+\mathcal{L}_{4}+\mathcal{L}_{2, \mathrm{~g} \chi \mathrm{SB}}^{*}, \\
\mathcal{L}_{2, \mathrm{~g} \chi \mathrm{SB}}^{*}= & -\frac{F_{\pi}^{2}}{32}\left\{-\left(\chi_{\mathrm{p}}^{ \pm}+\chi_{\mathrm{p}}^{0}\right) \operatorname{Tr} \boldsymbol{\nabla} U \cdot \nabla U^{\dagger}\right. \\
& +\left(\chi_{\mathrm{p}}^{ \pm}-\chi_{\mathrm{p}}^{0}\right) \operatorname{Tr} \boldsymbol{\nabla} U \tau_{3} \cdot \nabla U^{\dagger} \tau_{3} \\
& +\left(2 \mathcal{M}_{+}^{2}+\chi_{\mathrm{s}}^{ \pm}+\chi_{\mathrm{s}}^{0}\right) \operatorname{Tr}(U-1)\left(U^{\dagger}-1\right) \\
& \left.-\left(2 \mathcal{M}_{-}^{2}+\chi_{\mathrm{s}}^{ \pm}-\chi_{\mathrm{s}}^{0}\right) \operatorname{Tr}(U-1) \tau_{3}\left(U^{\dagger}-1\right) \tau_{3}\right\} .
\end{aligned}
$$

Here, $\chi_{\mathrm{s}, \mathrm{p}}^{ \pm}$and $\chi_{\mathrm{s}, \mathrm{p}}^{0}$ are functionals of the low-energy Sand $\mathrm{P}$-wave pion-nucleon scattering lengths and volumes, $b_{0,1}$ and $c_{0,1}$, respectively [22. They express the influence of the medium on the charged and neutral pion fields, $\pi^{ \pm}$and $\pi^{0}$. Furthermore, they depend on the densities of the neutron and proton distributions, $\rho_{\mathrm{n}}$ and $\rho_{\mathrm{p}}$, of the environment. One can check that the Lagrangian (6) - in the linear approximation - generates the well-known field equations $\left(\partial_{\mu} \partial^{\mu}+m_{\pi^{ \pm, 0}}^{2}+\hat{\Pi}^{ \pm, 0}\right) \pi^{ \pm, 0}=0[22$, where the polarization operators $\hat{\Pi}^{ \pm, 0}$ have the schematic form

$$
\hat{\Pi}^{ \pm, 0}=\chi_{\mathrm{s}}^{ \pm, 0}+\nabla \chi_{\mathrm{p}}^{ \pm, 0} \cdot \nabla .
$$

The medium-modified Lagrangian (6) can be rewritten in the following way

$$
\begin{aligned}
& \mathcal{L}^{*} \equiv \mathcal{L}_{2}^{*}+\mathcal{L}_{4}+\mathcal{L}_{\chi \mathrm{SB}}^{*}+\Delta \mathcal{L}^{*}, \\
& \mathcal{L}_{2}^{*}=\frac{F_{\pi}^{2}}{16}\left\{\operatorname{Tr} \partial_{0} U \partial_{0} U^{\dagger}-\left(1-\chi_{\mathrm{p}}^{0}\right) \operatorname{Tr} \nabla U \cdot \nabla U^{\dagger}\right\},
\end{aligned}
$$

1 This is manifest when the isospin-breaking term is rewritten as in Eq. (11). Because of $U U^{\dagger}=1$, Rathske's first term, the pion-mass term, is compatible with the usual choice of explicit chiral symmetry breaking proportional to $\operatorname{Tr}\left(U+U^{\dagger}-2\right)$ 13 .

${ }^{2}$ From now on an asterix indicates a density-dependent quantity.

$$
\begin{aligned}
\mathcal{L}_{\chi \mathrm{SB}}^{*}=- & \frac{F_{\pi}^{2} m_{\pi^{0}}^{2}}{16}\left(1+\frac{\chi_{\mathrm{s}}^{0}}{m_{\pi^{0}}^{2}}\right) \operatorname{Tr}(U-1)\left(U^{\dagger}-1\right), \\
\Delta \mathcal{L}^{*}=\frac{F_{\pi}^{2}}{32}\{ & \left(\chi_{\mathrm{p}}^{ \pm}-\chi_{\mathrm{p}}^{0}\right)\left[\operatorname{Tr}\left(\tau_{i} \boldsymbol{\nabla} U\right) \cdot \operatorname{Tr}\left(\tau_{i} \boldsymbol{\nabla} U^{\dagger}\right)\right. \\
& \left.-\operatorname{Tr}\left(\tau_{3} \boldsymbol{\nabla} U\right) \cdot \operatorname{Tr}\left(\tau_{3} \boldsymbol{\nabla} U^{\dagger}\right)\right] \\
- & \left(2 \mathcal{M}_{-}^{2}+\chi_{\mathrm{s}}^{ \pm}-\chi_{\mathrm{s}}^{0}\right)\left[\operatorname{Tr}\left(\tau_{i} U\right) \operatorname{Tr}\left(\tau_{i} U^{\dagger}\right)\right. \\
& \left.\left.-\operatorname{Tr}\left(\tau_{3} U\right) \operatorname{Tr}\left(\tau_{3} U^{\dagger}\right)\right]\right\}
\end{aligned}
$$

where $\mathcal{L}_{\chi \mathrm{SB}}^{*}$ is the chiral-symmetry-breaking term and $\Delta \mathcal{L}^{*}$ is the isospin-breaking term, separately. Note that in the absence of $\Delta \mathcal{L}^{*}$ the Lagrangian (8) reduces to the mediummodified Lagrangian presented in Ref. [19].

In general, the strong isospin breaking in the baryonic sector is generated by two effects: an explicit isospinbreaking in the mesonic sector (in the Lagrangian this term is proportional to $\mathcal{M}_{-}^{2}$ ) and the isospin-dependent influence of the medium on each of the pion fields, $\chi_{\mathrm{p}}^{ \pm}-$ $\chi_{\mathrm{p}}^{0}$ and $\chi_{\mathrm{s}}^{ \pm}-\chi_{\mathrm{s}}^{0}$. For simplicity and in order to concentrate on the effects due to explicit isospin breaking in the mesonic sector, we will consider from now on only isospinsymmetric nuclear matter:

$$
\begin{aligned}
& \chi_{\mathrm{p}}^{ \pm}=\chi_{\mathrm{p}}^{0} \equiv \chi_{\mathrm{p}}=\frac{4 \pi c_{0} \rho / \eta}{1+4 g^{\prime} \pi c_{0} \rho / \eta}, \\
& \chi_{\mathrm{s}}^{ \pm}=\chi_{\mathrm{s}}^{0} \equiv \chi_{\mathrm{s}}=-4 \pi \eta\left(b_{0}\right)_{\mathrm{eff}} \rho .
\end{aligned}
$$

Here $\eta=1+m_{\pi^{0}} / m_{\mathrm{N}}$ is a kinematical factor, $m_{\mathrm{N}}=$ $938 \mathrm{MeV}$ is the nucleon mass, $g^{\prime}$ is a correlation parameter, $c_{0}$ is the $\mathrm{P}$-wave isoscalar pion-nucleon scattering volume, $\left(b_{0}\right)_{\text {eff }}$ is the corresponding effective $\mathrm{S}$-wave isoscalar scattering length in nuclear matter, and $\rho$ is the density of the surrounding medium 22. Moreover, we will limit the investigation to homogenous nuclear matter, $\rho_{\mathrm{p}}=\rho_{\mathrm{n}}=$ $\rho / 2=$ const, and to the evaluation of the neutron-proton mass difference in nuclear matter, $\Delta m_{\mathrm{np}}^{*}=m_{\mathrm{n}}^{*}-m_{\mathrm{p}}^{*}$.

To finish this section, we remark that the approach used here should be distinguished from the method used in Refs. [26,27,28]. In these papers, all meson properties like the masses, decay constants, etc. were assumed to be density-dependent and taken from a microscopic model of the meson dynamics. Here, the density-dependence is parameterized in terms of phenomenological input, more precisely the effective scattering length $\left(b_{0}\right)_{\text {eff }}$ and volume $c_{0}$.

The Lagrangian (6) is of course only one representative of a larger class of Skyrme-type models with e.g. higherorder terms or vector-meson degrees of freedom which, rooted in a hadronic language, do allow for a simultaneous description of nucleon properties and interactions. However, the present choice with its scale-independent dimensionless Skyrme parameter $e$ can be considered as the simplest, but also as a generic one, with a minimal number of derivatives and model-parameters, that incorporates explicitly strong isospin breaking, stable solitons, and medium modifications. 


\section{Classical solitonic solutions}

Due to the isospin-breaking term of the Lagrangian (8), the third component of the isovector field is singled out in the field equations:

$$
\partial_{\mu} \frac{\partial \mathcal{L}^{*}}{\partial \partial_{\mu} \boldsymbol{\Phi}}-\frac{\partial \mathcal{L}^{*}}{\partial \boldsymbol{\Phi}}=0 .
$$

Treating the (strong) isospin-breaking as a small perturbation, we can distinguish between a non-perturbed baryonic background with $m_{\pi^{ \pm}}^{2}=m_{\pi^{0}}^{2}$ and perturbative contributions proportional to the very tiny strong-interaction induced part of $m_{\pi^{ \pm}}^{2}-m_{\pi^{0}}^{2} \neq 0$. The non-perturbed system has the usual time-independent classical (baryon number $B=1)$ solutions $\boldsymbol{\Phi}(t=0, \boldsymbol{r})=\boldsymbol{\phi}(\boldsymbol{r})$ of the isospinsymmetric Lagrangian, whereas a perturbation around the classical background $\phi(\boldsymbol{r})$ can be absorbed $e$.g. by a timedependent modification.

We follow Rathske [21] by using for this purpose the separation ansatz

$$
\boldsymbol{\Phi}(t, \boldsymbol{r})=\mathcal{T}(t) \boldsymbol{\phi}(\boldsymbol{r})
$$

where a manifest isospin-breaking (with respect to the $3 \mathrm{rd}$ isospin-axis) is encoded in the time-dependent iso-rotation matrix $\mathcal{T}$ :

$$
\mathcal{T}(t)=\left(\begin{array}{rrr}
\cos a^{*} t & \sin a^{*} t & 0 \\
-\sin a^{*} t & \cos a^{*} t & 0 \\
0 & 0 & 1
\end{array}\right)
$$

Here $a^{*}$ is a density-dependent global constant 4 which would vanish in the absence of the small perturbation $\Delta m_{\pi}=m_{\pi^{ \pm}}-m_{\pi^{0}}$, since then the system would reduce to the non-perturbed configuration $\lim _{\Delta m_{\pi} \rightarrow 0} \mathcal{L}^{*}=\mathcal{L}_{\mathrm{NP}}^{*}$.

The ansatz (14) together with (15), when inserted into the Lagrangian (8) and after spatial integration, allows to cancel the non-perturbative shift (IB-NP) proportional to $m_{\pi^{ \pm}}^{2}-m_{\pi^{0}}^{2}$ that results from inserting the non-perturbative (NP) background $\boldsymbol{\phi}(\boldsymbol{r})$ of the isospin-symmetric case into the isospin-breaking part (IB) of the Lagrange function against the isospin-breaking term $(\mathrm{IB}-\mathcal{T})$ that results from the time-dependent rotation by the $\mathcal{T}$ matrix:

$$
\begin{aligned}
& L^{*}=\int \mathcal{L}^{*} \mathrm{~d}^{3} r=-M_{\mathrm{NP}}^{*}-\mathcal{M}_{-}^{2} \Lambda_{-}^{*}+\frac{a^{* 2}}{2} \Lambda^{*} \\
& \equiv \int \mathcal{L}_{\mathrm{NP}}^{*} \mathrm{~d}^{3} r+\int \mathcal{L}_{\mathrm{IB}-\mathrm{NP}}^{*} \mathrm{~d}^{3} r+\int \mathcal{L}_{\mathrm{IB}-\mathcal{T}}^{*} \mathrm{~d}^{3} r .
\end{aligned}
$$

Here $M_{\mathrm{NP}}^{*}[\phi]$ is the isospin-symmetric mass of the nonperturbed configuration. The moment-of-inertia type quantities $\Lambda^{*}=\Lambda^{*}[\phi]$ and $\Lambda_{-}^{*}=\Lambda_{-}^{*}[\phi]$, belonging to the $\mathcal{T}$ rotation and the non-perturbative shift, respectively, are functionals of the pion fields. The global parameter $a^{*}$

\footnotetext{
${ }^{3}$ The explicit form of Eq. (13) can be found in Ref. 21 for the free space case. Note that the Skyrme term is omitted there.

4 Note that a sign change of $t$ can be compensated by a sign change of the angular velocity $a^{*}$.
}

serves here as a constraint parameter, whereas in Ref. 21 - under the neglect of stabilizing higher-order terms - the ansatz (14) also extremized the remaining action. Thus the value of $a^{* 2}$ is fixed here by the following condition which implies the cancellation between the IB-NP and IB$\mathcal{T}$ parts in the Lagrange function:

$$
a^{* 2}=2 \mathcal{M}_{-}^{2} \frac{\Lambda_{-}^{*}}{\Lambda^{*}} .
$$

As the iso-rotation matrix $\mathcal{T}$ is chosen in such a way that - at the classical level and after spatial integration - the isospin-breaking is rotated out of the system, the classical field equations (13) effectively reduce to the nonperturbed isospin-symmetric case:

$$
\partial_{\mu} \frac{\partial \mathcal{L}^{*}}{\partial \partial_{\mu} \boldsymbol{\Phi}}-\frac{\partial \mathcal{L}^{*}}{\partial \boldsymbol{\Phi}}=0 \Rightarrow \frac{\delta M_{\mathrm{NP}}^{*}[\boldsymbol{\phi}]}{\delta \boldsymbol{\phi}}=0 .
$$

In general, one should consider non-spherical timeindependent field configurations $\phi(\boldsymbol{r})$ if the density gradients of the surrounding nuclear environment are large. In this case, the static field configuration acquires a $\theta$ dependence 20, i.e. $\phi=\phi(|\boldsymbol{r}|, \theta)$, even if isospin-breaking terms are absent. The above presented "rotation" procedure has the advantage that it remains applicable for very non-uniform density-profile-dependent cases, in other words, for finite nuclei. But as stated in Sec. 2, only the case of an isospin-symmetric homogeneous nuclear environment with a constant density is considered here. Thus the use of a spherically symmetric static configuration $\phi(\boldsymbol{r})=(\boldsymbol{r} / r) F_{\pi} F(r) / 2$ is still appropriate. Under this hedgehog ansatz the terms defined in (16) are given by

$$
\begin{aligned}
M_{\mathrm{NP}}^{*}= & \pi \int_{0}^{\infty}\left\{\frac{F_{\pi}^{2}}{2}\left(1-\chi_{\mathrm{p}}\right)\left(F_{r}^{2}+\frac{2 S^{2}}{r^{2}}\right)\right. \\
& +\frac{2}{e^{2}}\left(2 F_{r}^{2}+\frac{S^{2}}{r^{2}}\right) \frac{S^{2}}{r^{2}} \\
& \left.+F_{\pi}^{2}\left(m_{\pi^{0}}^{2}+\chi_{\mathrm{s}}\right)(1-\cos F)\right\} r^{2} \mathrm{~d} r, \\
\Lambda^{*}= & \frac{2 \pi}{3} \int_{0}^{\infty}\left\{F_{\pi}^{2}+\frac{4}{e^{2}}\left(F_{r}^{2}+\frac{S^{2}}{r^{2}}\right)\right\} S^{2} r^{2} \mathrm{~d} r, \\
\Lambda_{-}^{*}= & \frac{2 \pi}{3} F_{\pi}^{2} \int_{0}^{\infty} S^{2} r^{2} \mathrm{~d} r,
\end{aligned}
$$

where $F_{r} \equiv \mathrm{d} F(r) / \mathrm{d} r$. Also the abbreviation $S \equiv \sin F(r)$ is used here.

The corresponding field equations (18) do not explicitly depend on the isospin index and therefore reduce to the simple radial form that is used in this work to determine the profile function $F(r)$ :

$$
\begin{aligned}
& \frac{F_{\pi}^{2}}{2}\left(1-\chi_{\mathrm{p}}\right)\left(F_{r r}+\frac{2}{r} F_{r}-\frac{\sin 2 F}{r^{2}}\right) \\
& +\frac{2}{e^{2}}\left(\frac{2 \sin ^{2} F}{r^{2}} F_{r r}+\frac{\sin 2 F}{r^{2}}\left(F_{r}^{2}-\frac{\sin ^{2} F}{r^{2}}\right)\right) \\
& -\frac{F_{\pi}^{2}}{2}\left(m_{\pi^{0}}^{2}+\chi_{\mathrm{s}}\right) \sin F=0 .
\end{aligned}
$$


Ulf-G. Meißner et al.: Neutron-proton mass difference in nuclear matter

Here $F_{r r}$ stands for the second derivative of the profile function $F(r)$ with respect to the radial coordinate $r$.

If the Skyrme term were omitted, the condition (17) would reduce to $a^{* 2}=m_{\pi^{ \pm}}^{2}-m_{\pi^{0}}^{2}$ and the results of the work 21 would be reproduced in a natural way. However in this case, due to the then profile-independent value of the parameter $a^{*}$, the density dependence of the isospinbreaking effects would be lost 5 . From this point of view, the presence of a higher-order term in the Lagrangian is crucial, not only for its stabilization role, but also for the description of the density-dependence of the isospinbreaking in the nuclear environment. Of course, there is a model-dependence due to the nature of the stabilizing term. We reiterate that the Skyrme term is the most simplest choice, with the least number of derivatives and model-constants, that guarantees the existence of stable baryons. In fact, the chiral order of the Skyrme term is the same as the one of the strong isospin-breaking term of chiral perturbation theory.

To finish this section, let us state the expectation that the above mentioned shift induced by the isospin-breaking terms and the compensating isospin-breaking rotation will be manifest in the quantized theory, although they are constrained to cancel each other at the classical level.

\section{Quantization and the strong part of $\Delta \mathrm{m}_{\mathrm{np}}^{*}$}

The standard quantization procedure of the Skyrme model [12] requires time-dependent rotations in space, $\mathcal{R}(t)$, and isospin-space, $\mathcal{I}(t)$ :

$$
\begin{aligned}
U(t, \boldsymbol{r}) & =\exp \left(i \frac{2}{F_{\pi}} \boldsymbol{\tau} \cdot \boldsymbol{\Phi}(t, \boldsymbol{r})\right) \\
\hookrightarrow U^{\prime}\left(t, \boldsymbol{r}^{\prime}\right) & =\exp \left(i \frac{2}{F_{\pi}} \boldsymbol{\tau} \cdot \mathcal{I}(t) \boldsymbol{\Phi}\left(t, \mathcal{R}^{-1}(t) \boldsymbol{r}\right)\right)
\end{aligned}
$$

where $\boldsymbol{\Phi}(t, \boldsymbol{r})$ is defined in Eq. (14). The rotations $\mathcal{R}(t)$ and iso-rotations $\mathcal{I}(t)$ correspond to standard collective zero-energy modes of the classical soliton, whereas the matrix $\mathcal{T}$ of Eq. (14) describes a constrained rotational mode with respect to the classical soliton. The $\mathrm{SO}(3)$ matrices, $\mathcal{I}(t)$ and $\mathcal{R}(t)$, satisfy the conditions

$$
\dot{\mathcal{I}}_{i k}(t) \mathcal{I}_{k j}^{-1}(t)=\varepsilon_{i j l} \omega_{l}, \quad \dot{\mathcal{R}}_{i k}^{-1}(t) \mathcal{R}_{k j}(t)=-\varepsilon_{i j l} \Omega_{l},
$$

where $\omega_{l}$ and $\Omega_{l}$ are the angular velocities of the isospin and spatial rotations, respectively, and the 'dot' symbolizes a time derivative. Under these rotations and the constraint (17) the corresponding Lagrange function $L^{*}=$ $\int \mathcal{L}^{*} d^{3} r$ takes the form

$$
L^{*}=-M_{\mathrm{NP}}^{*}+\frac{\Lambda^{*}}{2}\left[(\boldsymbol{\omega}-\boldsymbol{\Omega})^{2}+2 a^{*}\left(\omega_{3}-\Omega_{3}\right)\right] .
$$

Note that the third components of the angular velocities couple to the constrained angular velocity $a^{*}$. Finally, the

\footnotetext{
5 The isospin-breaking effects resulting from the pion-mass variation in the nuclear medium are beyond the scope of the present model.
}

usual Legendre transformation utilizing the canonical-conjugated operators $\hat{T}_{i}=\partial_{\omega_{i}} L^{*}, \hat{J}_{i}=\partial_{\Omega_{i}} L^{*}$ of $\omega_{i}$ and $\Omega_{i}$, respectively, leads to the quantum Hamiltonian:

$$
\begin{aligned}
\hat{H}^{*} & =M_{\mathrm{NP}}^{*}+\frac{\hat{\boldsymbol{T}}_{\mathbf{1}}{ }^{2}}{2 \Lambda^{*}}+\frac{\hat{\boldsymbol{T}}_{\mathbf{2}}{ }^{2}}{2 \Lambda^{*}}+\frac{\left(\hat{\boldsymbol{T}}_{\mathbf{3}}-\Lambda^{*} a^{*}\right)^{2}}{2 \Lambda^{*}} \\
& =M_{\mathrm{NP}}^{*}+\mathcal{M}_{-}^{2} \Lambda_{-}^{*}+\frac{\hat{\boldsymbol{T}}^{2}}{2 \Lambda^{*}}-a^{*} \hat{T}_{3} .
\end{aligned}
$$

There does not exist a canonical-conjugated momentum of the angular velocity $a^{*}$ since this is a constrained quantity. The mass-shift term due to the explicit isospin-breaking, the second term of Eq. (26), reappears - compared with Eq. (16) or Eq. (25) - after applying the constraint (17) to the induced term $\left(\Lambda^{*} / 2\right) a^{* 2}$. Moreover, note that the isospin breaking in the mesonic sector is manifest at the quantum level by the coefficient of the third component of the isospin operator, see the fourth term of Eq. (26). Consequently, by evaluating the quantum Hamiltonian (26) between appropriate nucleon states one can isolate the strong part of the neutron-proton mass difference in the nuclear medium as 6

$$
m_{\mathrm{n}}^{*(\text { strong })}-m_{\mathrm{p}}^{*(\text { strong })}=\Delta m_{\mathrm{np}}^{*(\text { strong })}=a^{*} .
$$

This term is enhanced in comparison to the tiny mass shift $\mathcal{M}_{-}^{2} \Lambda_{-}^{*}$, since the latter is quadratic and not linear in the small parameter $a^{*}$.

Finally, let us discuss the scaling under the expansion in the number of colors $N_{c}$ of QCD, the underlying theory of strong interactions. From the viewpoint of the nonrelativistic quark model, the mass difference of a large $N_{c}$ neutron (with $\left(N_{c}+1\right) / 2 d$-quarks and $\left(N_{c}-1\right) / 2$ $u$-quarks) and a large $N_{c}$ proton (with $\left(N_{c}+1\right) / 2 u$ quarks and $\left(N_{c}-1\right) / 2 d$-quarks) is expected to scale as $m_{d}-m_{u} \sim N_{c}^{0}$. Let us compare this with our Eq. (27): As usual, we start with the assumption that $F_{\pi} \sim 1 / e \sim$ $\sqrt{N_{c}}$, such that the soliton mass $M_{\mathrm{NP}}^{*}$, the moment-ofinertia $\Lambda^{*}$ and also $\Lambda_{-}^{*}$ scale as $\mathcal{O}\left(N_{c}^{1}\right)$. Assuming that $\mathcal{M}_{-}$scales as $\mathcal{M}_{+}$, namely as $\mathcal{O}\left(N_{c}^{0}\right), 7$ the constrained constant $a^{*}$ (see Eq. (17)) and the strong neutron-proton mass difference (27) is of the same order $\mathcal{O}\left(N_{c}^{0}\right)$. Therefore, the $N_{c}$ scaling of Eq. (26) is as it should be: the non-perturbative isospin-symmetric mass term $M_{\mathrm{NP}}^{*}$ and the non-perturbative (via isospin-breaking induced) mass shift $\mathcal{M}_{-}^{2} \Lambda_{-}^{*}$ scale both as $\mathcal{O}\left(N_{c}^{1}\right)$; the quantization term $\hat{\boldsymbol{T}}^{2} /\left(2 \Lambda^{*}\right)$ scales as $\mathcal{O}\left(N_{c}^{-1}\right)$, as expected for the quantization of zero-modes of a soliton [12] finally the isospin-

\footnotetext{
${ }^{6}$ Note that the sign of $a^{*}$ is now fixed by the known sign of the strong nucleon-proton mass difference in free space.

7 Note that the $N_{c}$ scaling of the SU(3) low-energy constant $L_{7} \sim(1 / 48) F_{\pi}^{2} / m_{\eta^{\prime}}^{2} \sim \mathcal{O}\left(N_{c}^{2}\right)$ [23] would actually imply that $\mathcal{M}_{-}^{2}$ should scale as $\mathcal{O}\left(N_{c}^{1}\right)$ (see (4) ), since the $\eta^{\prime}$ would become a Goldstone boson in the limit $N_{c} \rightarrow \infty$ (i.e., the $\eta^{\prime}$ mass scales as $\left.m_{\eta^{\prime}} \sim \sqrt{1 / N}\right)$. For $N_{c}=3$, however, the prefactor is very tiny, such that the above assumed $\mathcal{O}\left(N_{c}^{0}\right)$ behavior of $\mathcal{M}_{-}^{2}=\left(m_{\pi^{ \pm}}^{2}-m_{\pi^{0}}^{2}\right) / 2$ is the more natural choice for the strong pion mass difference.
} 
breaking term $a^{*} \hat{T}_{3}$ resulting from the constrained isorotation (with the enhancement by $\Lambda^{*} \sim N_{c}$ relative to the quantized isospin, see the first line of Eq. (26)) scales as $\mathcal{O}\left(N_{c}^{0}\right)$.

\section{Electromagnetic form factors and the electromagnetic part of $\Delta \mathrm{m}_{\mathrm{np}}^{*}$}

The electric (E) and magnetic (M) form factors of the nucleon are defined through the expressions

$$
\begin{aligned}
& G_{\mathrm{E}}^{*}\left(\boldsymbol{q}^{2}\right)=\frac{1}{2} \int \mathrm{d}^{3} r e^{i \boldsymbol{q} \cdot \boldsymbol{r}} j^{0}(r), \\
& G_{\mathrm{M}}^{*}\left(\boldsymbol{q}^{2}\right)=\frac{1}{2} m_{\mathrm{N}} \int \mathrm{d}^{3} r e^{i \boldsymbol{q} \cdot \boldsymbol{r}}[\boldsymbol{r} \times \boldsymbol{j}(r)],
\end{aligned}
$$

where $\boldsymbol{q}^{2}$ is the squared momentum transfer. Furthermore, $j^{0}$ and $\boldsymbol{j}$ correspond to the time and space components of the properly normalized sum of the baryonic current $B_{\mu}^{*}$ and the third component of the isovector current $\boldsymbol{V}_{\mu}^{*}$ of the Skyrme model, i.e.

$$
\begin{aligned}
B_{\mu}^{*}= & \frac{1}{24 \pi^{2}} \varepsilon_{\mu \nu \alpha \beta} \operatorname{Tr} L^{\nu} L^{\alpha} L^{\beta} \\
V_{\mu}^{(3) *}= & \frac{i}{16} \operatorname{Tr}\left\{\tau_{3}\left(-F_{\pi}^{2} C_{\mu} L_{\mu}+\frac{1}{e^{2}}\left[L^{\nu},\left[L_{\mu}, L_{\nu}\right]\right]\right)\right\} \\
& +(L \rightarrow R) \\
C_{\mu}= & \left\{\begin{array}{cc}
1 & , \quad \mu=0 \\
1-\chi_{\mathrm{p}}, & \mu=1,2,3 .
\end{array}\right.
\end{aligned}
$$

Here $\varepsilon_{\mu \nu \alpha \beta}$ is the totally antisymmetric tensor in four dimensions and the definition $R_{\mu}=U \partial_{\mu} U^{\dagger}$ is used. Note that the index $\mu$ in Eq. (30) is not summed over. By evaluating these current operators between appropriate nucleon states, one obtains the density-dependent electromagnetic form factors of the nucleon [26, 27, 28, 29, 30].

Although the isoscalar $(\mathrm{S})$ electric and magnetic form factors of the nucleon

$$
\begin{aligned}
& G_{\mathrm{E}}^{\mathrm{S} *}=-\frac{1}{\pi} \int_{0}^{\infty} F_{r} \sin ^{2} F j_{0}(q r) \mathrm{d} r, \\
& G_{\mathrm{M}}^{\mathrm{S} *}=-\frac{m_{\mathrm{N}}}{\pi \Lambda^{*}} \int_{0}^{\infty} F_{r} \sin ^{2} F r^{2} \frac{j_{1}(q r)}{q r} \mathrm{~d} r
\end{aligned}
$$

do not explicitly depend on the density, there still exists an implicit medium modification caused by the density dependence of the profile function $F$. This holds also for the isovector $(\mathrm{V})$ electric form factor

$$
\begin{aligned}
G_{\mathrm{E}}^{\mathrm{V} *}=\frac{\pi}{3 \Lambda^{*}} & \int_{0}^{\infty}\left\{F_{\pi}^{2}+\frac{4}{e^{2}}\left(F_{r}^{2}+\frac{\sin ^{2} F}{r^{2}}\right)\right\} \\
& \times \sin ^{2} F r^{2} j_{0}(q r) \mathrm{d} r .
\end{aligned}
$$

The isovector magnetic form factor, however, has both explicit and implicit density dependences:

$$
\begin{gathered}
G_{\mathrm{M}}^{\mathrm{V} *}=\frac{2 \pi m_{\mathrm{N}}}{3} \int_{0}^{\infty}\left\{F_{\pi}^{2}\left(1-\chi_{\mathrm{p}}\right)+\frac{4}{e^{2}}\left(F_{r}^{2}+\frac{\sin ^{2} F}{r^{2}}\right)\right\} \\
\times \sin ^{2} F r^{2} \frac{j_{1}(q r)}{q r} \mathrm{~d} r
\end{gathered}
$$

The medium-dependent form factors of the proton and neutron are defined as $G_{\mathrm{E}, \mathrm{M}}^{\left(\begin{array}{c}\mathrm{p} \\ \mathrm{n}\end{array}\right) *}=G_{\mathrm{E}, \mathrm{M}}^{\mathrm{S} *} \pm G_{E, M}^{\mathrm{V} *}$ with the normalization conditions $G_{\mathrm{E}}^{\mathrm{p} *}(0)=1, G_{\mathrm{E}}^{\mathrm{n} *}(0)=0, G_{\mathrm{M}}^{\mathrm{p} *}(0)$ $=\mu_{\mathrm{p}}^{*}, G_{\mathrm{M}}^{\mathrm{n} *}(0)=\mu_{\mathrm{n}}^{*}$, where $\mu_{\mathrm{p}}^{*}$ and $\mu_{\mathrm{n}}^{*}$ are the in-medium magnetic moments of the proton and neutron, respectively. Note that the magnetic moments are measured in terms of the nuclear Bohr magneton (abbreviated as n.m. in Tab. 11) with the free-space nucleon mass $m_{\mathrm{N}}$. Therefore, $m_{\mathrm{N}}$ and not $m_{\mathrm{N}}^{*}$ is used in Eqs. (28), (32) and (34).

Finally, for calculating the electromagnetic part of the neutron-proton mass difference, one can apply the formula

$$
\begin{aligned}
\Delta m_{\mathrm{np}}^{*(\mathrm{EM})}=-\frac{4 \alpha}{\pi} \int_{0}^{\infty} \mathrm{d} q\left\{G_{\mathrm{E}}^{\mathrm{S} *}\left(\boldsymbol{q}^{2}\right) G_{\mathrm{E}}^{\mathrm{V} *}\left(\boldsymbol{q}^{2}\right)\right. \\
\left.\quad-\frac{\boldsymbol{q}^{2}}{2 m_{\mathrm{N}}^{2}} G_{\mathrm{M}}^{\mathrm{S} *}\left(\boldsymbol{q}^{2}\right) G_{\mathrm{M}}^{\mathrm{V} *}\left(\boldsymbol{q}^{2}\right)\right\},
\end{aligned}
$$

see e.g. Ref. 31]. Here $\alpha=\mathrm{e}^{2} / 4 \pi \approx 1 / 137$ is the electromagnetic fine structure constant (with e the elementary charge). Note also that the right-hand-side of this equation, after Eqs. (32) to (34) are inserted, does not depend on the value of the nucleon mass and moreover that $\Delta m_{\mathrm{np}}^{*(\mathrm{EM})}$ scales as $\mathcal{O}\left(N_{c}^{0}\right)$ like $\Delta m_{\mathrm{np}}^{* \text { (strong) }}$.

\section{Input parameters of the model}

As input for the free mass of the neutral pion we take the PDG-value 32]: $m_{\pi^{0}}=134.977 \mathrm{MeV}$. This choice induces the values $F_{\pi}=108.11 \mathrm{MeV}$ and $e=4.826$, if one insists on reproducing the empirical (isospin-averaged) masses of the nucleon and delta, $m_{\mathrm{N}}=938 \mathrm{MeV}$ and $M_{\Delta}=$ $1232 \mathrm{MeV}$, in free space $(\rho=0)$ and without isospin breaking term $\left(\mathcal{M}_{-}=0\right)$. The small differences to the values $F_{\pi}=108 \mathrm{MeV}$ and $e=4.84$ of Ref. [13] are caused by the value $m_{\pi^{0}}=138 \mathrm{MeV}$ used there.

The mass $m_{\pi^{ \pm}}$of the charged pions, actually only its strong contribution 8 to $\Delta m_{\pi}=m_{\pi^{ \pm}}-m_{\pi^{0}}$, is extracted as a variational parameter from the fit to the empirical value $\Delta m_{\mathrm{np}}^{(\mathrm{Exp})}=1.29 \mathrm{MeV}$ in free space. To do this, we first calculate the electromagnetic part of the neutronproton mass difference in free space in analogy to the formula (35), $\Delta m_{\mathrm{np}}^{(\mathrm{EM})}=-0.68 \mathrm{MeV}$. This is in good agreement with the result of Ebrahim and Savci 33 obtained long ago. Model calculations, which include $\pi, \rho$

\footnotetext{
8 The electromagnetic contribution to $m_{\pi^{ \pm}}$is not considered throughout this paper.
} 
Table 1. Masses and magnetic moments of the nucleons in nuclear matter with a density $\rho=\lambda \rho_{0}$, where $\rho_{0}=0.5 m_{\pi^{0}}^{3}$ is the saturation density of ordinary nuclear matter.

\begin{tabular}{ccccc}
\hline$\lambda$ & $m_{\mathrm{p}}^{*}[\mathrm{MeV}]$ & $m_{\mathrm{n}}^{*}[\mathrm{MeV}]$ & $\mu_{\mathrm{p}}^{*}[\mathrm{n} . \mathrm{m}]$. & $\mu_{\mathrm{n}}^{*}[\mathrm{n} . \mathrm{m}]$. \\
\hline 0 & 937.4 & 938.7 & 1.99 & -1.27 \\
0.2 & 861.9 & 863.2 & 1.86 & -1.13 \\
0.4 & 796.6 & 797.9 & 1.75 & -1.02 \\
0.6 & 739.6 & 740.9 & 1.65 & -0.92 \\
0.8 & 689.5 & 690.8 & 1.56 & -0.83 \\
1.0 & 645.2 & 646.4 & 1.48 & -0.75 \\
\hline
\end{tabular}

and $\omega$ mesons, also lead to similar results [34. Moreover, these results also are consistent with the value $\Delta m_{\mathrm{np}}^{(\mathrm{EM})}=$ $(-0.76 \pm 0.30) \mathrm{MeV}$ estimated from the Cottingham formula in Ref. 31. Finally, applying

$$
m_{\left(\begin{array}{l}
\mathrm{p} \\
\mathrm{n}
\end{array}\right)}=m_{\left(\begin{array}{c}
\mathrm{p} \\
\mathrm{n}
\end{array}\right)}^{(\text {strong })} \mp \frac{1}{2} \Delta m_{\mathrm{np}}^{(\mathrm{EM})}
$$

we find the following estimate of the neutron-proton mass difference due to the strong interactions: $\Delta m_{\mathrm{np}}^{\text {(strong) }}=$ $\Delta m_{\mathrm{np}}^{(\mathrm{Exp})}-\Delta m_{\mathrm{np}}^{(\mathrm{EM})}=1.97 \mathrm{MeV}$ which is fitted in the present model by adjusting (the strong part of) $\Delta m_{\pi}$, i.e. the quantities $\mathcal{M}_{+}$and $\mathcal{M}_{-}$of Eq. (5). In contrast to the sizable value of $\Delta m_{\mathrm{np}}^{\text {(strong) }}$ the pion-mass difference due to strong interactions is very small9: $\Delta m_{\pi}^{\text {(strong) }} \simeq 0.04 \mathrm{MeV}$, compatible with the results of Ref. [31.

Finally, the parameters of the pion self-energy are taken from Ref. 22, Table 6.2: $g_{0}^{\prime}=1 / 3, c_{0}=0.21 m_{\pi}^{-3}$, and $\left(b_{0}\right)_{\text {eff }}=-0.024 m_{\pi}^{-1}$. The latter two are based on the tabulated isospin $1 / 2$ and $3 / 2 \mathrm{~S}_{1 / 2}, \mathrm{P}_{1 / 2}$ and $\mathrm{P}_{3 / 2}$ pionnucleon scattering lengths from Ref. [35] which are still compatible with modern calculations in the framework of chiral perturbation theory, see e.g. Tab. 2 of Ref. 36].

\section{Results and discussion}

The medium-dependent effective masses and magnetic moments of the nucleons are presented in Tab. 1 for a couple of values of the nuclear density. As expected, the effective masses decrease with increasing density of the nuclear medium. The most dominant contribution to these changes comes from the explicit medium $\left(1-\chi_{\mathrm{p}}\right)$ factor in the expression of the mass functional $M_{\mathrm{NP}}^{*}$ of the nonperturbed system (19).

Of course, it is well established that the effective mass of the nucleon decreases in the medium [37,38. However, the in-medium behavior of the neutron-proton mass difference is still under debate. Our results for the mass difference due to the strong interaction, $\Delta m_{\mathrm{np}}^{* \text { (strong) }}$, and electromagnetic interaction, $\Delta m_{\mathrm{np}}^{*(\mathrm{EM})}$, are presented in Fig. 1 and Fig. 2, respectively.

\footnotetext{
${ }^{9}$ In Ref. 21, where the Skyrme term is absent, this value is even equal to $\approx 0.01 \mathrm{MeV}$.
}

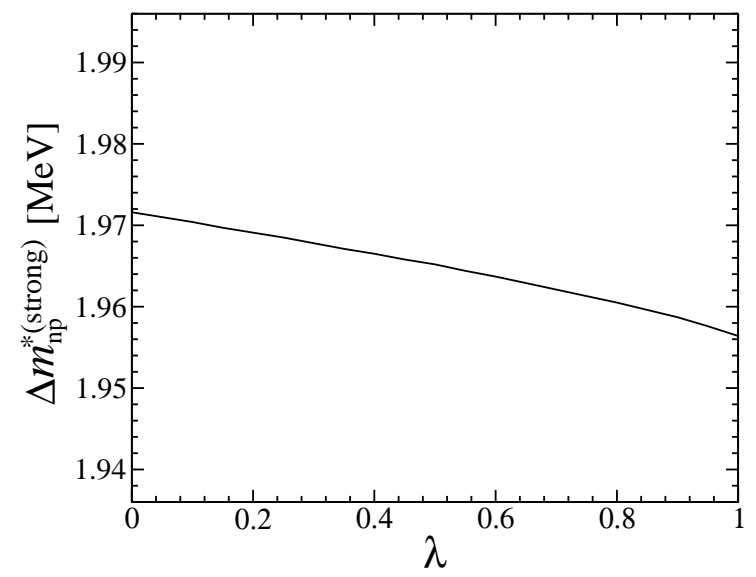

Fig. 1. Density dependence of the strong part of the neutronproton mass difference. The abscissa represents $\lambda=\rho / \rho_{0}$, while the ordinate represents $\Delta m_{\mathrm{np}}^{* \text { (strong) }}$ in units of $\mathrm{MeV}$.

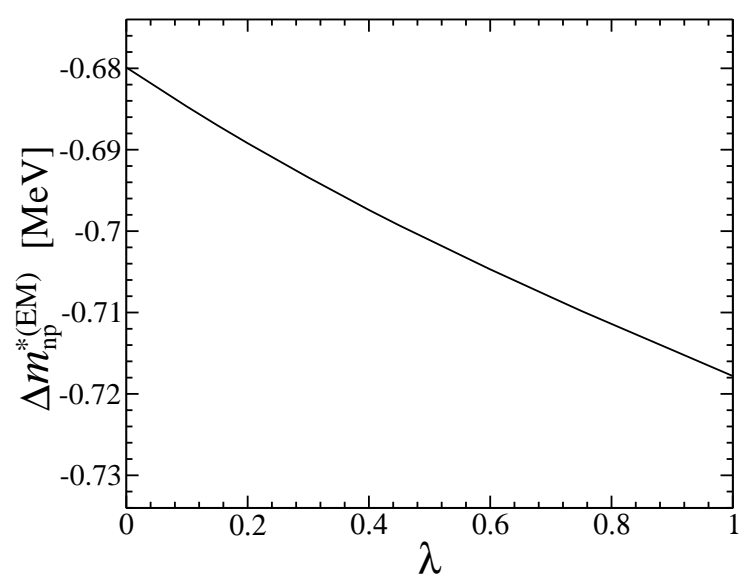

Fig. 2. Density dependence of the electromagnetic part of the neutron-proton mass difference. The abscissa represents $\lambda=$ $\rho / \rho_{0}$, while the ordinate represents $\Delta m_{\mathrm{np}}^{*(\mathrm{EM})}$ in units of $\mathrm{MeV}$.

It can be seen that both parts, the strong and electromagnetic one, have an almost linear dependence on the medium density and decrease with increasing the density. Note, however, that the absolute values of the changes are very small: i.e., the strong part of $\Delta m_{\mathrm{np}}^{*}$ is almost flat (see Fig. 1), while the change in the electromagnetic part is slightly more pronounced (see Fig. 2). The difference in their behavior follows from the fact that $\Delta m_{\mathrm{np}}^{* \text { (strong) }}$ has no explicit dependence on the medium functionals (see Eqs. (17) and (27)), whereas $\Delta m_{\mathrm{np}}^{*(\mathrm{EM})}$ (35) explicitly depends on the $\mathrm{P}$-wave medium functional via the isovector magnetic form factor (34). However, there is a chance that the corresponding shifts may be more pronounced in isospin-asymmetric matter, due to the pertinent inmedium functionals in the isospin breaking term of the Lagrangian $\Delta \mathcal{L}^{*}$ (see Eq. (11) ) and the additional dependence of the medium functionals on the difference of the neutron-proton (distribution) densities of the surrounding environment, $\delta \rho=\rho_{\mathrm{n}}-\rho_{\mathrm{p}}$. 


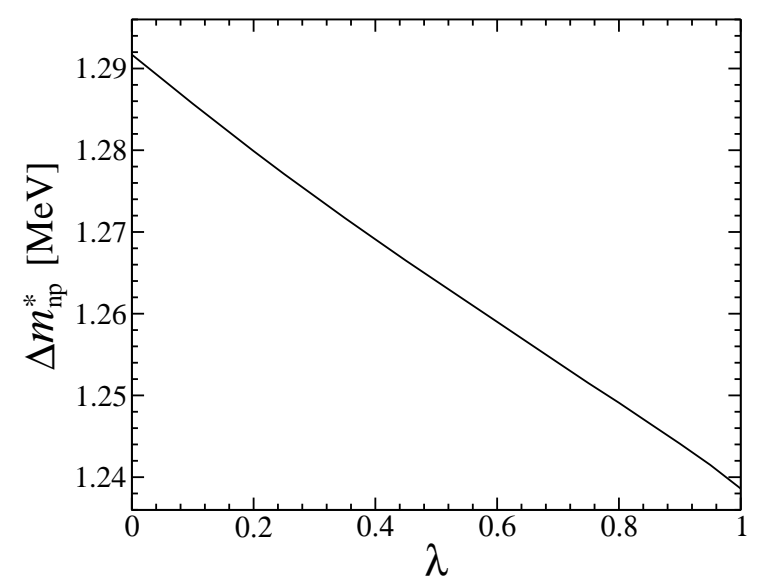

Fig. 3. Density dependence of the total neutron-proton mass difference. The abscissa represents $\lambda=\rho / \rho_{0}$, while the ordinate represents $\Delta m_{\mathrm{np}}^{*}$ in units of $\mathrm{MeV}$.

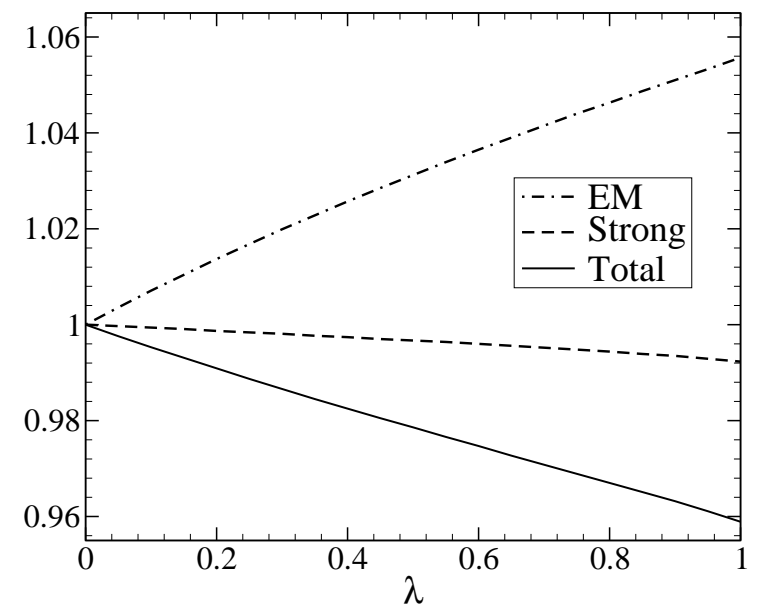

Fig. 4. Density dependence of the following neutronproton mass differences, normalized to their free space values: $\Delta m_{\mathrm{np}}^{*(\mathrm{EM})} / \Delta m_{\mathrm{np}}^{(\mathrm{EM})}, \Delta m_{\mathrm{np}}^{*(\text { strong) }} / \Delta m_{\mathrm{np}}^{\text {(strong) }}, \Delta m_{\mathrm{np}}^{*} / \Delta m_{\mathrm{np}}$ plotted by the dash-dotted, dashed and solid curves, respectively. The abscissa represents $\lambda=\rho / \rho_{0}$, while the ordinate represents the three dimensionless ratios.

In Fig. 3 the total neutron-proton mass difference $\Delta m_{\mathrm{np}}^{*}$ in the nuclear medium is shown as function of the nuclear density. For convenience, we present also, normalized to their free space values, the neutron-proton mass differences $\Delta m_{\mathrm{np}}^{*(\mathrm{EM})} / \Delta m_{\mathrm{np}}^{(\mathrm{EM})}, \Delta m_{\mathrm{np}}^{* \text { (strong) }} / \Delta m_{\mathrm{np}}^{\text {(strong) }}$, and $\Delta m_{\mathrm{np}}^{*} / \Delta m_{\mathrm{np}}$, in Fig. [4 by the dash-dotted, dashed and solid curves, respectively.

From Fig. 4 one can see that the change in the neutronproton mass difference is only $\sim 4 \%$ at normal nuclear matter density and that the total value $\Delta m_{\mathrm{np}}^{*}$ decreases in the nuclear medium as function of the density. This result is in qualitative agreement with the result of the quarkmeson coupling model [7. At the quantitative level, however, they differ: the absolute value of the quantity $\Delta m_{\mathrm{np}}^{*}$, within the present approach, decreases about $0.05 \mathrm{MeV}$ at normal nuclear matter density (see Fig. 3), while in Ref. [7] the decrease is about $0.85 \mathrm{MeV}$. One can conclude that in the Skyrme model, contrary to the result of the work [7], $\Delta m_{\mathrm{np}}^{*}$ remains positive - even at high densities - if the surrounding environment has an isospin-symmetric structure. Note that in isospin-asymmetric matter this picture may change.

The density dependence of the neutron-proton mass difference is approximately linear and can be parameterized as

$$
\Delta m_{\mathrm{np}}^{*} \simeq 1.3 \mathrm{MeV}-C \rho / \rho_{0} .
$$

Our calculation shows that $C \simeq 0.05 \mathrm{MeV}$, which is almost negligible in comparison with the calculation [6] in the framework of QCD sum rules, $C \simeq 1.1 \div 1.7 \mathrm{MeV}$.

\section{Summary and outlook}

We have investigated the isospin-breaking effects for nucleons embedded into an isospin-symmetric nuclear environment. In order to calculate these effects, a mediummodified version of the Skyrme model, which also takes into account explicit isospin breaking, has been proposed. Our calculations within this framework show that, with increasing density, the total neutron-proton mass difference as well as its strong and its electromagnetic part, separately, decrease only by a very small amount.

A generalization of the present approach to finite nuclei, i.e. to calculations of the type presented in Ref. [20], would allow to evaluate the isospin-breaking effects in mirror nuclei with the following qualifications:

- the strong part of the neutron-proton mass difference would separately depend on the $\mathrm{S}$ - and $\mathrm{P}$-wave medium functionals $\chi_{\mathrm{s}, \mathrm{p}}^{ \pm}, \chi_{\mathrm{s}, \mathrm{p}}^{0}$ and, consequently, would strongly depend on the parameterization of the latter ones [22];

- the medium functionals would explicitly depend on the neutron-proton density distributions $\chi_{\mathrm{s}, \mathrm{p}}^{ \pm, 0}=\chi_{\mathrm{s}, \mathrm{p}}^{ \pm, 0}\left(\rho_{\mathrm{n}}+\right.$ $\left.\rho_{\mathrm{p}}, \rho_{\mathrm{n}}-\rho_{\mathrm{p}}\right)[22$, such that the main property of mirror nuclei

$$
{ }_{Z}^{A} \mathbf{M}_{N} \Leftrightarrow{ }_{\tilde{Z}}^{A} \widetilde{\mathbf{M}}_{\tilde{N}}={ }_{N}^{A} \widetilde{\mathbf{M}}_{Z}
$$

could be taken into account in a natural way;

- additional effects could arise due to an $\omega$-dependence of the polarization operators $\hat{\Pi}^{ \pm, 0}=\hat{\Pi}^{ \pm, 0}(\omega, \boldsymbol{k})$ in Eq. (77), e.g. from the Weinberg-Tomozawa 39, 40 and the so-called range terms [41, see also Refs. 42, 43.

- the evaluation of local isospin-breaking effects, according to the nucleon-position $\boldsymbol{R}$ as measured from the center of the nucleus [20], would lead to additional possibilities.

The consequences of these additional modifications are the subject of future studies.

10 Note that in the present work, the polarization operator, because of the form of Eq. (6) and the isospin symmetry of the surrounding environment, is simply given by the static formula $\hat{\Pi}^{ \pm, 0}=\hat{\Pi}\left(\omega=m_{\pi^{0}}, \boldsymbol{k}\right)$. 
We thank Norbert Kaiser for a useful correspondence. The work of U.T.Y. was supported by the Alexander von Humboldt Foundation. A.M.R. thanks Prof. V. E. Kravtsov for hospitality during his stay at the ICTP, Italy, the Deutscher Akademischer Austausch Dienst (DAAD) for a travel scholarship to Jülich, Germany, and the Forschungszentrum Jülich for hospitality. Partial financial support from the EU Integrated Infrastructure Initiative Hadron Physics Project (contract number RII3CT-2004-506078), by the DFG (TR 16, "Subnuclear Structure of Matter") and BMBF (research grant 06BN411) is gratefully acknowledged.

\section{References}

1. J. A. Nolen and J. P. Schiffer, Ann. Rev. Nucl. Part. Sci. 19, 471 (1969).

2. E. M. Henley and G. Krein, Phys. Rev. Lett. 62, 2586 (1989).

3. T. Hatsuda, H. Hogaasen and M. Prakash, Phys. Rev. C 42, 2212 (1990).

4. T. Hatsuda, H. Høgaasen and M. Prakash, Phys. Rev. Lett. 66, 2851 (1991) [Erratum-ibid. 69, 1290 (1992)].

5. C. Adami and G. E. Brown, Z. Phys. A 340, 93 (1991).

6. T. Schäfer, V. Koch and G. E. Brown, Nucl. Phys. A 562, 644 (1993).

7. K. Saito and A. W. Thomas, Phys. Lett. B 335, 17 (1994) arXiv:nucl-th/9405009.

8. U.-G. Meißner and H. Weigel, Phys. Lett. B 267, 167 (1991).

9. M. Fiolhais, C. Christov, T. Neuber, M. Bergmann and K. Goeke, Phys. Lett. B 269, 43 (1991).

10. T. H. R. Skyrme, Proc. Roy. Soc. Lond. A 260, 127 (1961).

11. T. H. R. Skyrme, Nucl. Phys. 31, 556 (1962).

12. G. S. Adkins, C. R. Nappi and E. Witten, Nucl. Phys. B 228, 552 (1983).

13. G. S. Adkins and C. R. Nappi, Nucl. Phys. B 233, 109 (1984).

14. I. Zahed and G. E. Brown, Phys. Rep. 142, 1 (1986).

15. A. Jackson, A. D. Jackson and V. Pasquier, Nucl. Phys. A 432, 567 (1985).

16. U. B. Kaulfuß and U.-G. Meißner, Phys. Rev. D 31, 3024 (1985).

17. C. Hajduk, B. Schwesinger, Nucl. Phys. A 453, 620 (1986).

18. T. Otofuji, S. Saito, M. Yasuno, H. Kanada and T. Kurihara, Prog. Theor. Phys. 78, 527 (1987).

19. A. Rakhimov, M. M. Musakhanov, F. C. Khanna and U. T. Yakhshiev, Phys. Rev. C 58, 1738 (1998) arXiv:nucl-th/9609049.

20. U. T. Yakhshiev, M. M. Musakhanov, A. M. Rakhimov, U.-G. Meißner and A. Wirzba, Nucl. Phys. A 700, 403 (2002) arXiv:nucl-th/0109008.

21. E. Rathske, Z. Phys. A 331, 499 (1988).

22. T. Ericson and W. Weise, Pions and Nuclei (Clarendon, Oxford, 1988).

23. J. Gasser and H. Leutwyler, Nucl. Phys. B 250, 465 (1985).

24. L. N. Epele, H. Fanchiotti, C. A. Garcia Canal and R. Mendez Galain, Phys. Rev. D 39, 1473 (1989).

25. P. Jain, R. Johnson, N. W. Park, J. Schechter and H. Weigel, Phys. Rev. D 40, 855 (1989).

26. U.-G. Meißner, Phys. Rev. Lett. 62, 1013 (1989).

27. U.-G. Meißner, Phys. Lett. B 220, 1 (1989).
28. U.-G. Meißner, Nucl. Phys. A 503, 801 (1989).

29. M. M. Musakhanov, A. M. Rakhimov, U. T. Yakhshiev and Z. Kanokov, Phys. Atom. Nucl. 62, 1845 (1999) [Yad. Fiz. 62, 1988 (1999)].

30. U. T. Yakhshiev, U.-G. Meißner and A. Wirzba, Eur. Phys. J. A 16, 569 (2003) arXiv:nucl-th/0211055.

31. J. Gasser and H. Leutwyler, Phys. Rep. 87, 77 (1982).

32. W. M. Yao et al. [Particle Data Group], J. Phys. G 33, 1 (2006).

33. A. Ebrahim and M. Savci, Phys. Lett. B 189, 343 (1987).

34. N. Kaiser and U.-G. Meißner, Nucl. Phys. A 489, 671 (1988).

35. G. Höhler, Pion-nucleon scattering, Landolt-Börnstein (ed. H. Schopper), (Springer, Berlin, 1983), Vol. 9b2, p. 279.

36. N. Fettes and U.-G. Meißner, Nucl. Phys. A 676, 311 (2000) arXiv:hep-ph/0002162.

37. J. P. Jeukenne, A. Lejeune and C. Mahaux, Phys. Rep. 25, 83 (1976).

38. C. Mahaux, P. F. Bortignon, R. A. Broglia, and C. H. Dasso, Phys. Rep. 120, 1 (1985).

39. S. Weinberg, Phys. Rev. Lett. 17, 616 (1966).

40. Y. Tomozawa, Nuovo Cim. A 46, 707 (1966).

41. J. Delorme, M. Ericson and T. E. O. Ericson, Phys. Lett. B 291, 379 (1992).

42. V. Thorsson and A. Wirzba, Nucl. Phys. A 589, 633 (1995) arXiv:nucl-th/9502003.

43. U.-G. Meißner, J. A. Oller and A. Wirzba, Annals Phys. 297, 27 (2002) arXiv:nucl-th/0109026. 